\title{
Efficient computation of joint fractional Fourier domain signal representation
}

\author{
Lutfiye Durak, ${ }^{1, *}$ Ahmet Kemal Özdemir, ${ }^{2}$ and Orhan Arikan ${ }^{3}$ \\ ${ }^{1}$ Department of Electronics and Communications Engineering, Yildiz Technical University, Besiktas, \\ Istanbul 34349, Turkey \\ ${ }^{2}$ WesternGeco AS, Schlumberger House/Solbraavein 23, Asker 1383, Norway \\ ${ }^{3}$ Department of Electrical Engineering, Bilkent University, Ankara 06800, Turkey \\ *Corresponding author: lutfiye@ieee.org
}

Received August 3, 2007; revised December 19, 2007; accepted January 9, 2008;

posted January 10, 2008 (Doc. ID 86062); published February 21, 2008

\begin{abstract}
A joint fractional domain signal representation is proposed based on an intuitive understanding from a timefrequency distribution of signals that designates the joint time and frequency energy content. The joint fractional signal representation (JFSR) of a signal is so designed that its projections onto the defining joint fractional Fourier domains give the modulus square of the fractional Fourier transform of the signal at the corresponding orders. We derive properties of the JFSR, including its relations to quadratic time-frequency representations and fractional Fourier transformations, which include the oblique projections of the JFSR. We present a fast algorithm to compute radial slices of the JFSR and the results are shown for various signals at different fractionally ordered domains. (C) 2008 Optical Society of America
\end{abstract}

OCIS codes: $070.0070,070.2575$.

\section{INTRODUCTION}

One of the major areas of research in time-frequency signal processing is the design of novel time-frequency representations that are utilized to analyze and process nonstationary signals $[1,2]$. As time-frequency distributions do not always convey desirable qualifications in every application, the demand for powerful signal representations has led to a substantial amount of research on the design of $2 \mathrm{D}$ signal representations defined by alternative variables other than time and frequency. Joint time-scale representations, which have attracted much interest especially in the fields of sonar and image processing, constitute one of the earliest examples of this type of representation. Other popular choices of joint variables include higher derivatives of the instantaneous phase of signals for radar and sonar problems [3,4]; dispersive time-shifts for wave propagation problems and analogs of quantum mechanical quantities such as spin, angular momentum, and radial momentum [5]; and scale-hyperbolic time, warped time-frequency, and warped time-scale. Such signal representations have been derived by using two alternative approaches that are both based on the operator theory: the variables are associated with either Hermitian operators as in [2] or unitary operators as in [5]. Similarly, a joint fractional signal representation has been derived in a mathematical framework by associating Hermitian fractional operators to fractional Fourier transform (FrFT) variables constituting the joint distribution [6].

The fractional Fourier domains are the set of all domains interpolating between time and frequency $[7,8]$. The associated transform, FrFT with order $a$ transforms a signal into the $a$ th-order fractional Fourier domain and the $a$ th-order FrFT of $x(t)$ is given by [9]

$$
x_{a}(t) \equiv\left\{\mathcal{F}^{a} x\right\}(t)=\int B_{a}\left(t, t^{\prime}\right) x\left(t^{\prime}\right) \mathrm{d} t^{\prime}, \quad-2<a<2,
$$

where

$$
B_{a}\left(t, t^{\prime}\right)=\frac{e^{-j(\pi \operatorname{sgn}(a) / 4+\phi / 2)}}{|\sin \phi|^{1 / 2}} e^{j \pi\left(t^{2} \cot \phi-2 t t^{\prime} \csc \phi+t^{\prime 2} \cot \phi\right)}
$$

is the transformation kernel, $\phi=a(\pi / 2)$ and $\operatorname{sgn}()$ is the sign function.

The fractional Fourier domains corresponding to $a=0$ and 1 are the time and frequency domains, respectively. The FrFT with an order parameter $a=a_{0}$ transforms a signal into the $a_{0}$ th-order fractional Fourier domain, which is oriented by $\phi_{0}=a_{0} \pi / 2$ with respect to the time axis in the counterclockwise direction as illustrated in Fig. 1. The FrFT has found many applications in signal processing [10-16], optics, diffraction theory, optical propagation, and optical signal processing [17-20].

In this paper, we derive the joint fractional signal representation (JFSR), which designates the energy contents of signals in fractional Fourier domain variables instead of time and frequency. To this end, rather than using cumbersome mathematical equations based on operator theory, we extend our intuitive understanding from a time-frequency distribution, i.e., a function that designates joint time and frequency contents of signals. Then, we derive some important properties of the JFSR including its relation to quadratic time-frequency representations and fractional Fourier transformations, and present a simple formula for its oblique projections. We also present a fast algorithm to compute radial slices of the JFSR and numerically computed JFSRs of some synthetic signals. 


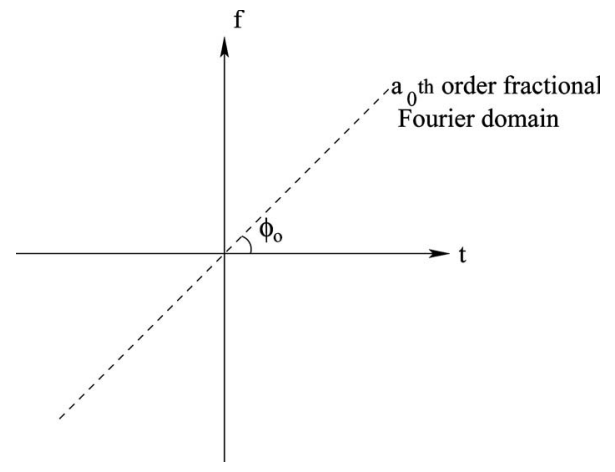

Fig. 1. $a_{0}$ th-order fractional Fourier domain makes an angle of $\phi_{0}=a_{0}(\pi / 2)$ on the time-frequency plane.

The outline of the paper is as follows. In Section 2, a concise derivation of JFSR is presented and some of its properties are provided. After presenting a fast computation algorithm in Section 3, JFSRs of some synthetic signals are numerically computed in Section 4. Finally, conclusions are drawn in Section 5.

\section{DISTRIBUTION OF SIGNAL ENERGY ON JOINT FRACTIONAL FOURIER DOMAINS}

One of the primary expectations from a time-frequency distribution $D_{x}(t, f)$ associated with a signal $x(t)$ is that it accurately represents the energy distribution of $x(t)$. It is desired that the signal energy at a frequency $f$ for a time instant $t$ is given by $D_{x}(t, f)$. Because of the uncertainty relationship between time and frequency domains, it is impossible to satisfy this pointwise energy density requirement. Therefore, one must usually be satisfied with a looser condition on the marginal densities

$$
\begin{aligned}
& \int D(t, f) \mathrm{d} f=|x(t)|^{2}, \\
& \int D(t, f) \mathrm{d} t=|X(f)|^{2},
\end{aligned}
$$

and integration of the distribution on the whole timefrequency plane

$$
\iint D(t, f) \mathrm{d} t \mathrm{~d} f=\|x\|^{2},
$$

where $X(f)$ is the Fourier transform of $x(t)$, and \|\| denotes the $L_{2}$ norm. One of the prominent energetic distributions that satisfies the desired relations [Eqs. (4) and (5)] is the Wigner distribution (WD), which is defined as [2]

$$
W_{x}(t, f)=\int x(t+\tau / 2) x^{*}(t-\tau / 2) e^{-j 2 \pi \tau f} \mathrm{~d} \tau .
$$

The WD of a signal can be roughly interpreted as an energy density of the signal, since it is real and covariant to time and frequency domain translations; and, moreover, signal energy in any extended time-frequency region can be determined by integrating $W_{x}(t, f)$ over that region. Another nice property of the WD is that its oblique projec- tions give the energy distribution with respect to the corresponding fractional Fourier domain [21]. Such properties and its ability to provide high time-frequency domain signal concentration make the WD attractive compared to other representations. Although the WD and its enhanced versions are useful in time-frequency analysis, in some applications such as signal design and synthesis it is more useful to have a time-fractional Fourier domain representation.

In this section, the JFSR is constructed using conditions similar to the ones given in Eqs. (3)-(5). The JFSR is then generalized to a cross JFSR, and some properties of the JFSR are investigated in detail.

\section{A. JFSR}

Let the JFSR of a signal be denoted by $E_{x}^{\mathbf{a}}(u, v)$ where a $=\left(a_{1}, a_{2}\right)$ denotes the orders of fractional Fourier domains $u$ and $v$, respectively. It is desired that the marginal densities satisfy

$$
\begin{gathered}
\int E_{x}^{\mathbf{a}}(u, v) \mathrm{d} v=\left|x_{a_{1}}(u)\right|^{2}, \\
\int E_{x}^{\mathbf{a}}(u, v) \mathrm{d} u=\left|x_{a_{2}}(v)\right|^{2},
\end{gathered}
$$

where $\left|x_{a_{1}}(u)\right|^{2}$ and $\left|x_{a_{2}}(v)\right|^{2}$ are the energy contents of the signal at the $a_{1}$ th and $a_{2}$ th fractional Fourier domains, respectively. Similar to Eq. (5), the overall integral on the $u-v$ plane is desired to be

$$
\iint E_{x}^{\mathbf{a}}(u, v) \mathrm{d} u \mathrm{~d} v=\|x\|^{2} .
$$

The conditions stated in Eqs. (7)-(9) make the JFSR a generalization of the WD, since the JFSR reduces to the WD when $\left(a_{1}, a_{2}\right)=(0,1)$.

To construct the distribution $E_{x}^{\mathbf{a}}(u, v)$ satisfying the conditions on the marginal densities and the total energy, we make use of the projection property of the WD [21]:

$$
\int W_{x}(u \cos \phi-v \sin \phi, u \sin \phi+v \cos \phi) \mathrm{d} v=\left|x_{a}(u)\right|^{2} .
$$

In Fig. 2, we observe that the value of the time-frequency distribution at a point $P$ contributes to the energy densities of the fractional Fourier domains $u$ and $v$ at points $u=u_{0}$ and $v=v_{0}$, respectively. Therefore, the JFSR can simply be formed by redistributing the WD so that

$$
E_{x}^{\mathbf{a}}(u, v)=C W_{x}(P(t(u, v), f(u, v)))
$$

where the coordinates $(u, v)$ and $(t, f)$ are related by

$$
\left[\begin{array}{cc}
\cos \phi_{1} & \sin \phi_{1} \\
\cos \phi_{2} & \sin \phi_{2}
\end{array}\right]\left[\begin{array}{l}
t \\
f
\end{array}\right]=\left[\begin{array}{l}
u \\
v
\end{array}\right],
$$

and $\phi_{i}=a_{i} \pi / 2$ for $i=1,2$. By using the total energy constraint [Eq. (9)], the constant $C$ in Eq. (11) is determined as 


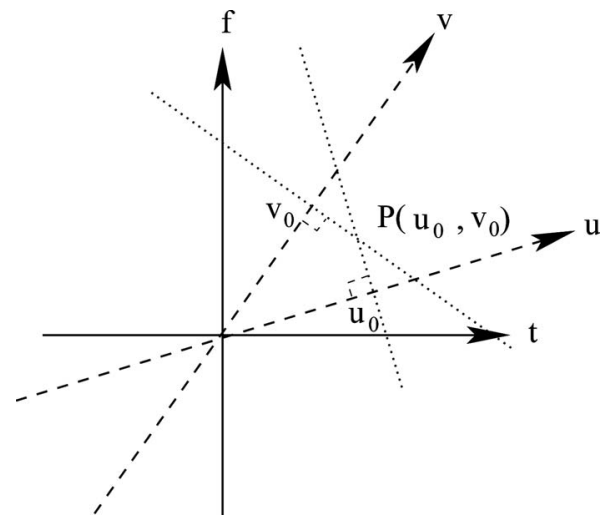

Fig. 2. Value of the time-frequency distribution at a point $P$ contributes to the energy densities of the fractional Fourier domains $u$ and $v$ at points $u=u_{0}$ and $v=v_{0}$, respectively.

$$
C=\left|\csc \left(\phi_{12}\right)\right|,
$$

where $\phi_{12}=\phi_{2}-\phi_{1}$. Thus, $E_{x}^{\mathbf{a}}(u, v)$ is explicitly given by

$$
\begin{aligned}
& \left|\csc \left(\phi_{12}\right)\right| \int x\left(\frac{u \sin \phi_{2}-v \sin \phi_{1}}{\sin \phi_{12}}+\tau / 2\right) x^{*} \\
& \quad \times\left(\frac{u \sin \phi_{2}-v \sin \phi_{1}}{\sin \phi_{12}}-\tau / 2\right) \\
& \quad \times \exp \left(-j 2 \pi \tau \frac{-u \cos \phi_{2}+v \cos \phi_{1}}{\sin \phi_{12}}\right) \mathrm{d} \tau .
\end{aligned}
$$

An equivalent and more compact form of $E_{x}^{\mathbf{a}}(u, v)$ can be obtained by using the rotation effect of the FrFT on the WD,

$$
W_{x_{a_{1}}}(u, v)=W_{x}\left(u \cos \phi_{12}-v \sin \phi_{12}, u \sin \phi_{12}+v \cos \phi_{12}\right),
$$

which verbally translates that WD of the $a_{1}$ th-order FrFT of a signal $x(t)$ is the same as the WD of the signal $x(t)$, which is rotated by $\phi_{1}$ radians in the clockwise direction in the time-frequency plane. Consequently, $E_{x}^{\mathbf{a}}(u, v)$ can be derived in terms of the fractionally Fourier transformed signal $x_{a_{1}}(t)$ as

$$
\begin{aligned}
E_{x}^{\mathbf{a}}(u, v)= & \int x_{a_{1}}\left(u+\frac{\tau \sin \phi_{12}}{2}\right) x_{a_{1}}^{*}\left(u-\frac{\tau \sin \phi_{12}}{2}\right) \\
& \times e^{-j 2 \pi\left(v-u \cos \phi_{12}\right) \tau} \mathrm{d} \tau,
\end{aligned}
$$

which has the same form as given in [6].

\section{B. Cross JFSR}

The JFSR can be generalized to define a cross JFSR of signals $x(t)$ and $y(t)$

$$
\begin{aligned}
E_{x y}^{\mathbf{a}}(u, v)= & \left|\csc \left(\phi_{12}\right)\right| W_{x y}(P(t(u, v), f(u, v))) \\
= & \int x_{a_{1}}\left(u+\frac{\tau \sin \phi_{12}}{2}\right) y_{a_{1}}^{*}\left(u-\frac{\tau \sin \phi_{12}}{2}\right) \\
& \times e^{-j 2 \pi\left(v-u \cos \phi_{12}\right) \tau} \mathrm{d} .
\end{aligned}
$$

Defining $E_{x}^{\mathbf{a}}(u, v)$ through its relation to the WD provides an easily interpretable definition of the JFSR of sig- nals. From the definition given in Eq. (15), it follows that JFSR is a quadratic distribution while it is not a timefrequency distribution. Therefore it belongs to a broader class than the familiar Cohen's class. Thus, its introduction to the nonstationary signal processing will bring new insights into the design, filtering, analysis, and synthesis of signals in many applications.

\section{Properties of the JFSR}

In this section, we investigate the properties of the JFSR. In the properties listed below, the joint fractional Fourier domains have the orders of $\mathbf{a}=\left(a_{1}, a_{2}\right)$ making angles of $\left(\phi_{1}, \phi_{2}\right)$ with respect to the time axis where $\phi_{i}=a_{i}(\pi / 2)$.

Property 1. The JFSR is a real distribution

$$
E_{x}^{\mathbf{a}}(u, v)=\left(E_{x}^{\mathbf{a}}\right) *(u, v) .
$$

Property 2. The orthogonal projections of the JFSR of a signal $x(t)$ onto $u$ and $v$ axes give the magnitude square of the FrFTs of the signal at orders associated with these axes, that is,

$$
\begin{aligned}
& \int E_{x}^{\mathbf{a}}(u, v) \mathrm{d} v=\left|x_{a_{1}}(u)\right|^{2}, \\
& \int E_{x}^{\mathbf{a}}(u, v) \mathrm{d} u=\left|x_{a_{2}}(v)\right|^{2} .
\end{aligned}
$$

Property 3. The area under the JFSR of a signal $x(t)$ gives the total signal energy

$$
\iint E_{x}^{\mathbf{a}}(u, v) \mathrm{d} u \mathrm{~d} v=\int|x(t)|^{2} \mathrm{~d} t,
$$

which follows from Property 2 and the unitarity of the FrFT.

Property 4. The JFSR and WD of a signal $x(t)$ are related as

$$
\begin{aligned}
E_{x}^{\mathbf{a}}(u, v)= & \left|\csc \phi_{12}\right| W_{x_{a_{1}}}\left(u, \frac{v-u \cos \phi_{12}}{\sin \phi_{12}}\right) \\
= & \left|\csc \phi_{12}\right| W_{x}\left(\frac{u \sin \phi_{2}-v \sin \phi_{1}}{\sin \phi_{12}},\right. \\
& \left.\times \frac{-u \cos \phi_{2}+v \cos \phi_{1}}{\sin \phi_{12}}\right) .
\end{aligned}
$$

Property 5. The JFSR and the FrFT of a signal $x(t)$ are related as

$$
E_{x_{a^{\prime}}}^{\mathbf{a}}(u, v)=E_{x}^{\mathbf{a}+a^{\prime}}(u, v),
$$

where $\mathbf{a}+a^{\prime}=\left(a_{1}+a^{\prime}, a_{2}+a^{\prime}\right)$.

Proof: By using Eq. (21) in Property 4, the JFSR of $x_{a}(t)$ can be written as

$$
\begin{aligned}
E_{x_{a^{\prime}}}^{\mathbf{a}}(u, v)= & \left|\csc \phi_{12}\right| W_{x_{a^{\prime}}}\left(\frac{u \sin \phi_{2}-v \sin \phi_{1}}{\sin \phi_{12}},\right. \\
& \left.\times \frac{-u \cos \phi_{2}+v \cos \phi_{1}}{\sin \phi_{12}}\right) .
\end{aligned}
$$

Then, by using the rotation property of the WD given in 
Eq. (14), the right-hand side of this expression is simplified to

$$
\begin{gathered}
E_{x_{a^{\prime}}}^{\mathbf{a}}(u, v)=\left|\csc \phi_{12}\right| W_{x}\left(u^{\prime}, v^{\prime}\right), \\
u^{\prime}=\frac{u \sin \left(\phi^{\prime}+\phi_{2}\right)-v \sin \left(\phi^{\prime}+\phi_{1}\right)}{\sin \phi_{12}}, \\
v^{\prime}=\frac{-u \cos \left(\phi^{\prime}+\phi_{2}\right)+v \cos \left(\phi^{\prime}+\phi_{1}\right)}{\sin \phi_{12}},
\end{gathered}
$$

which proves the property where $\phi^{\prime}=a^{\prime}(\pi / 2)$.

Property 6. Any oblique projection of JFSR of a signal $x(t)$ onto an oblique axis making an angle of $\phi$ is

$$
P_{\phi}\left[E_{x}^{\mathbf{a}}\right](r)=\left|x_{\left(a^{\prime}\right)}(r / M)\right|^{2}, \quad a^{\prime}=\frac{2 \phi^{\prime}}{\pi},
$$

where

$$
\begin{aligned}
& \phi^{\prime}=\arctan 2\left(\cos \phi_{1}+\cos \phi+\cos \phi_{2} \sin \phi, \sin \phi_{1} \cos \phi\right. \\
& \left.+\sin \phi_{2} \sin \phi\right), \\
& M=\left(1+\sin 2 \phi \cos \phi_{12}\right)^{1 / 2} .
\end{aligned}
$$

Proof: By the projection-slice theorem, the oblique projection of the JFSR of $x(t)$ at an angle $\phi$ is given by

$$
P_{\phi}\left[E_{x}^{\mathbf{a}}\right](r)=\int F_{x}(\zeta \cos \phi, \zeta \sin \phi) e^{-j 2 \pi \zeta r} \mathrm{~d} \zeta,
$$

where

$$
F_{x}^{\mathbf{a}}(\zeta, \eta)=\iint E_{x}^{\mathbf{a}}(u, v) e^{j 2 \pi(\zeta u+\eta v)} \mathrm{d} u \mathrm{~d} v,
$$

is the radial slice of the $2 \mathrm{D}$ inverse Fourier transform of the JFSR of $x(t)$. By using Eq. (21), the following expression can be obtained for $F_{x}^{(a)}(\zeta, \eta)$ in terms of the ambiguity function $A_{x}()$ of $x(t)$

$$
F_{x}^{\mathbf{a}}(\zeta, \eta)=A_{x}\left(\zeta \cos \phi_{1}+\eta \cos \phi_{2}, \zeta \sin \phi_{1}+\zeta \sin \phi_{2}\right) .
$$

Thus, the radial slice $F_{x}^{\mathbf{a}}(\zeta \cos \phi, \zeta \sin \phi)$ of $F_{x}^{\mathbf{a}}(\zeta, \eta)$ can be expressed as

$$
F_{x}^{\mathbf{a}}(\zeta \cos \phi, \zeta \sin \phi)=A_{x}\left(\zeta M \cos \phi^{\prime}, \zeta M \sin \phi^{\prime}\right),
$$

where $\phi^{\prime}$ and $M$ are as given in Eqs. (23) and (24), respectively. It is known that the radial slice of the ambiguity function of a signal $x(t)$ at the angle $\phi$ has the following relation to the $\left(a^{\prime}\right)$ th FrFT of the signal $x(t)$

$$
A_{x}\left(\zeta \cos \phi^{\prime}, \zeta \sin \phi^{\prime}\right)=\int\left|x_{\left(a^{\prime}\right)}(r)\right|^{2} e^{j 2 \pi \zeta r} \mathrm{~d} r .
$$

Then, the relation in Eq. (22) can be obtained by combining Eqs. (25), (28), and (29).

Property 7. Similar to the time-bandwidth product analysis on the joint time-frequency plane, the product of the spreads of signals in arbitrary fractional Fourier domains can also be defined. In [9], it has been shown that the product of the spreads of a signal $x$ ( ) in two arbitrary fractional Fourier domains $a_{1}$ and $a_{2}$ are bounded below by

$$
\sigma_{a_{1}} \sigma_{a_{2}} \geq \frac{\left|\sin \left[\pi\left(a_{1}-a_{2}\right) / 2\right]\right|}{4 \pi}
$$

where $\sigma_{a_{1,2}}$ is defined as follows:

$$
\sigma_{a_{1,2}}=\left[\int\left(u_{a_{1,2}}-\eta_{a_{1,2}}\right)^{2}\left|x_{a_{1,2}}\left(u_{a_{1,2}}\right)\right|^{2} \mathrm{~d} u_{a_{1,2}}\right]^{1 / 2} /\|x\|,
$$

$$
\eta_{a_{1,2}}=\left[\int u_{a_{1,2}}\left|x_{a_{1,2}}\left(u_{a_{1,2}}\right)\right|^{2} \mathrm{~d} u_{a_{1,2}}\right] /\|x\|^{2},
$$

where $u_{a_{1,2}}$ represents the $a_{1,2}$ th-order fractional Fourier domains. In [22], a tighter lower bound is derived for real signals, and it is shown that this bound actually corresponds to the product of fractional domain spreads of a Gaussian function. The analysis on the fractional domain spreads indicates that, as the joint parameters vary, the product of the spreads and consequently the area of the support of the signals on the joint fractional plane varies, and it diminishes to 0 as the joint fractional Fourier order parameters are equal to each other.

\section{FAST COMPUTATION OF THE JFSR}

In this section, we provide an efficient computation algorithm of the JFSR of a signal on arbitrary radial slices. Throughout the computations, we assume that the signal $x(t)$ is scaled to $x(t / s)$ before sampling, so that its WD is approximately confined into a circle of radius $\Delta_{x} / 2$. Here, if the time width and bandwidth of the signal is approximately $\Delta_{t}$ and $\Delta_{f}$, respectively, then the scaling parameter $s$ becomes $s=\left(\Delta_{f} / \Delta_{t}\right)^{1 / 2}$ providing a signal that has negligible energy outside the interval $\left[-\Delta_{x} / 2, \Delta_{x} / 2\right]$.

To compute the radial slice of the JFSR of a signal $x(t)$, we use the relation

$$
E_{x_{a}}^{\mathbf{a}}(r \cos \phi, r \sin \phi)=\left|\csc \phi_{12}\right| W_{x}\left(r \cos \phi^{\prime}, r \sin \phi^{\prime}\right),
$$

where

$$
\begin{aligned}
\phi^{\prime}= & \arctan \left(\cos \phi \cos \phi_{2}+\sin \phi \cos \phi_{1}, \cos \phi \sin \phi_{2}\right. \\
& \left.-\sin \phi \cos \phi_{1}\right),
\end{aligned}
$$

and $\phi_{1}$ and $\phi_{2}$ are the corresponding angles of the fractional Fourier domains $u$ and $v$ with respect to the time axis. It has been shown in [15] that the radial slice of the WD along the line $\left(r \cos \phi^{\prime}, r \sin \phi^{\prime}\right)$ is

$$
W_{x}\left(r \cos \phi^{\prime}, r \sin \phi^{\prime}\right)=\int x_{\left(a^{\prime}-1\right)}\left(\frac{-\lambda}{2}\right) x_{\left(a^{\prime}-1\right)}^{*}\left(\frac{\lambda}{2}\right) e^{-j 2 \pi r \lambda} \mathrm{d} \lambda .
$$


Therefore, Eqs. (33) and (35) can be used to construct the radial slice of $E_{x_{a}}^{\mathbf{a}}(u, v)$ as

$$
\begin{aligned}
E_{x_{a}}^{\mathbf{a}}(r \cos \phi, r \sin \phi)= & \left|\csc \phi_{12}\right| \int x_{\left(a^{\prime}-1\right)}\left(\frac{\lambda}{2}\right) x_{\left(a^{\prime}-1\right)}^{*}\left(\frac{-\lambda}{2}\right) \\
& \times e^{-j 2 \pi r \lambda} \mathrm{d} \lambda .
\end{aligned}
$$

If the double-sided bandwidth of $x_{\left(a^{\prime}-1\right)}$ is $\Delta_{x}$ and the time-bandwidth product is $N$, then the integral in Eq. (36) can be discretized by

$$
E_{x_{a}}^{\mathbf{a}}\left(r \Delta_{x} \cos \phi, r \Delta_{x} \sin \phi\right)=\frac{\left|\csc \phi_{12}\right|}{\Delta_{x}} \sum_{k=-N}^{N-1} q[k] e^{-\left(j 2 \pi r k / \Delta_{x}\right)},
$$

where $q[k]=x_{\left(a^{\prime}-1\right)}[k] x_{\left(a^{\prime}-1\right)}^{*}[-k]$ and $x_{\left(a^{\prime}-1\right)}[k]=x_{\left(a^{\prime}-1\right)}$ $\times\left(k /\left(2 \Delta_{x}\right)\right)$ is computed using the algorithm given in [23] with $O(N \log N)$ computational complexity.

As the relationship [Eq. (36)] depends on the FrFTs of the signal $x(t)$, computation of any $M$ uniformly spaced samples on the line segment $r \in\left[r_{i}, r_{f}\right]$ along the radial slice of $E_{x_{\alpha}}^{\mathbf{a}}\left(r \Delta_{x} \cos \phi, r \Delta_{x} \sin \phi\right)$ can be performed through the chirp- $z$ transform algorithm in $O((N+M) \log (N+M))$ computational complexity [24]. In Section 4, the results of the algorithm are presented for synthetic signals on various joint fractional Fourier planes.

\section{SIMULATIONS}

In this section, the JFSR of a chirp signal and a quadratic FM modulated signal that has a nonconvex timefrequency support on the time-frequency plane are evaluated for four different joint fractional Fourier order pairs of $\mathbf{a}=\left(a_{1}, a_{2}\right)$ as $(0,0.25),(0,0.5),(0,0.75)$, and $(0,1)$. Moreover, an application example, which makes use of the localization property of the JFSR is presented by employing the time-frequency component analyzer algorithm in [25].

For a chirp signal of $x(t)=\operatorname{rect}(t / 10) \exp \left(j 2 \pi\left(0.5 t^{2}+t\right)\right)$, the JFSRs of the four different $\mathbf{a}=\left(a_{1}, a_{2}\right)$ are presented in Fig. 3. The distribution given in Fig. 3(d) is the same as the WD of $x(t)$ because the fractional order parameters of the domains are $a_{1}=0, a_{2}=1$, corresponding to the time and frequency domains, respectively. As shown in Fig. 3, the supports of the chirp signal at various joint fractional Fourier planes remains linear. However, the localization of the resultant components varies, because the uncertainty relation of the fractional Fourier domains has a tighter lower-bound when compared to the time and frequency domains [9].

The JFSRs of a quadratic FM modulated signal $x(t)$ $=\exp \left(-\pi\left((t / 3)^{2}+0.3 j t^{3}\right)\right)$, which has a nonconvex timefrequency support on the time-frequency plane are presented in Fig. 4. The distribution given in Fig. 4(d) with fractional Fourier order pair $(0,1)$ is the same as the WD of $x(t)$. It is easier to observe the localization of the signal component that depends on the order of the joint fractional Fourier domains. By comparing Figs. 4(a) and 4(d), it will be noticed that the amount of cross-term interference is significantly less when the two fractional orders are closer to each other. We expect that these types of (a)

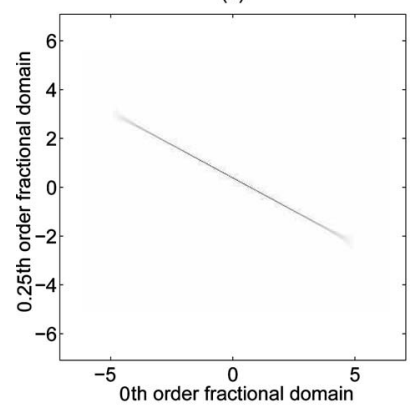

(c)

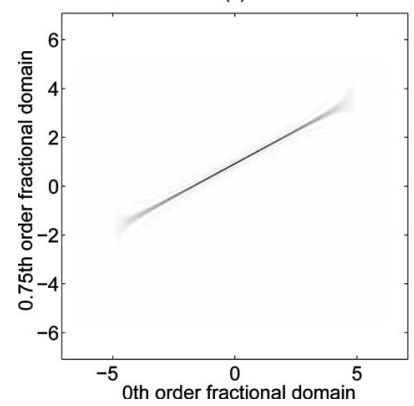

(b)

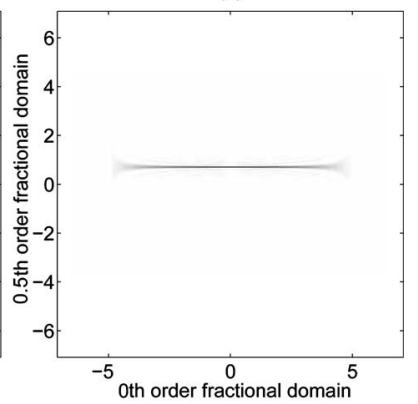

(d)

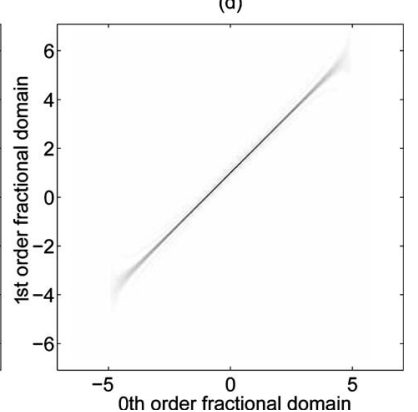

Fig. 3. JFSRs of $x(t)=\operatorname{rect}(t / 10) e^{j 2 \pi\left(0.5 t^{2}+t\right)}$ at joint fractional Fourier domains with orders (a) $\left(a_{1}, a_{2}\right)=(0,0.25)$, (b) $\left(a_{1}, a_{2}\right)$ $=(0,0.5),(\mathrm{c})\left(a_{1}, a_{2}\right)=(0,0.75),(\mathrm{d})\left(a_{1}, a_{2}\right)=(0,1)$.

properties of JFSR will facilitate the analysis and design of time-frequency distributions where the signal components have curved time-frequency supports.

We also compute the JFSR of a $2.5 \mathrm{~ms}$ echolocation pulse emitted by a large brown bat, eptesicus fuscus. The recorded signal is plotted in Fig. 5(a) and can be downloaded at [26]. The time axis in Fig. 5(a) has been scaled as discussed in [23]. Figures 5(b)-5(d) show the JFSR of the bat signal that has been evaluated for three different joint fractional Fourier order pairs of $\mathbf{a}=\left(a_{1}, a_{2}\right)$ as $(0,1)$, $(0,1.2)$, and $(0,1.3)$. The distribution given in Fig. $5(\mathrm{~b})$ is the same as the WD, because the fractional order parameters $a_{1}=0$ and $a_{2}=1$ correspond to the time and frequency domains, respectively. We see that the bat signal is composed of several components with nonconvex timefrequency supports. Therefore, the WD contains both inner and outer interference terms. In each of the plots, Figs. 5(c) and 5(d), one of the components in the bat signal has a very localized fractional frequency content. This is consistent with the fact that the uncertainty relation of the fractional Fourier domains has a tighter lower-bound when compared to the time and frequency domains [9].

Because of its localization property, the JFSR can be used to compute the instantaneous fractional frequency content of the analyzed signals. This will be illustrated on the signal shown in Fig. 6(a), which is one of the components extracted from the bat signal given in Fig. 5(a) by using the time-frequency component analyzer algorithm [25]. Figure 6(b) shows the WD of the component and Fig. 6(c) shows its JFSR corresponding to the fractional Fourier order pairs of $(0,1.2)$. In this domain, the analyzed component contains only a very narrow band of $a_{2}=1.2$ th-order fractional frequency. Here we extend the definition of instantaneous frequency to the fractional domain as follows: 

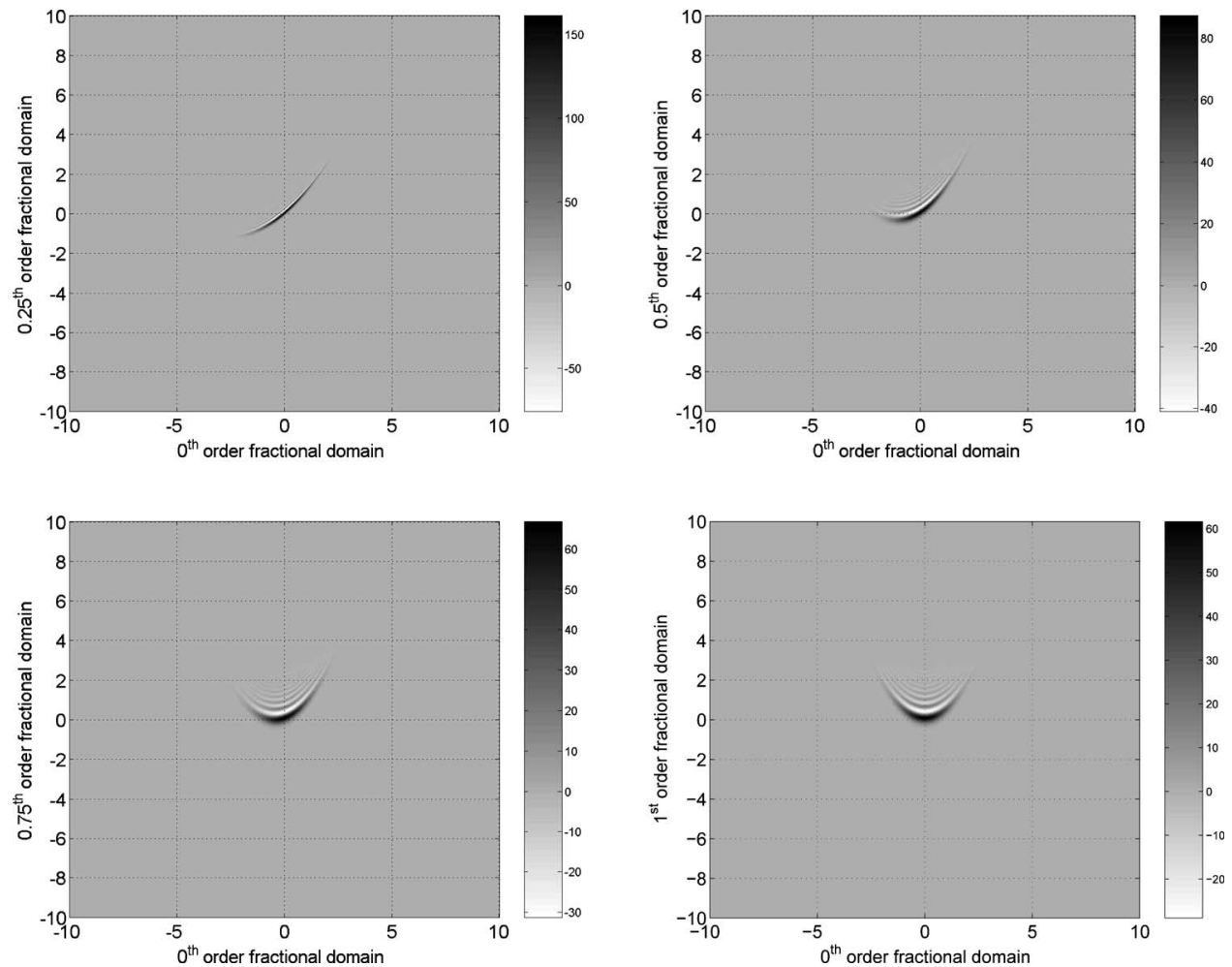

Fig. 4. JFSRs of $x(t)=e^{-\pi\left((t / 3)^{2}+0.3 j t^{3}\right)}$ at joint fractional Fourier domains with orders (a) $\left(a_{1}, a_{2}\right)=(0,0.25),(\mathrm{b})\left(a_{1}, a_{2}\right)=(0,0.5),(\mathrm{c})$ $\left(a_{1}, a_{2}\right)=(0,0.75),(\mathrm{d})\left(a_{1}, a_{2}\right)=(0,1)$.
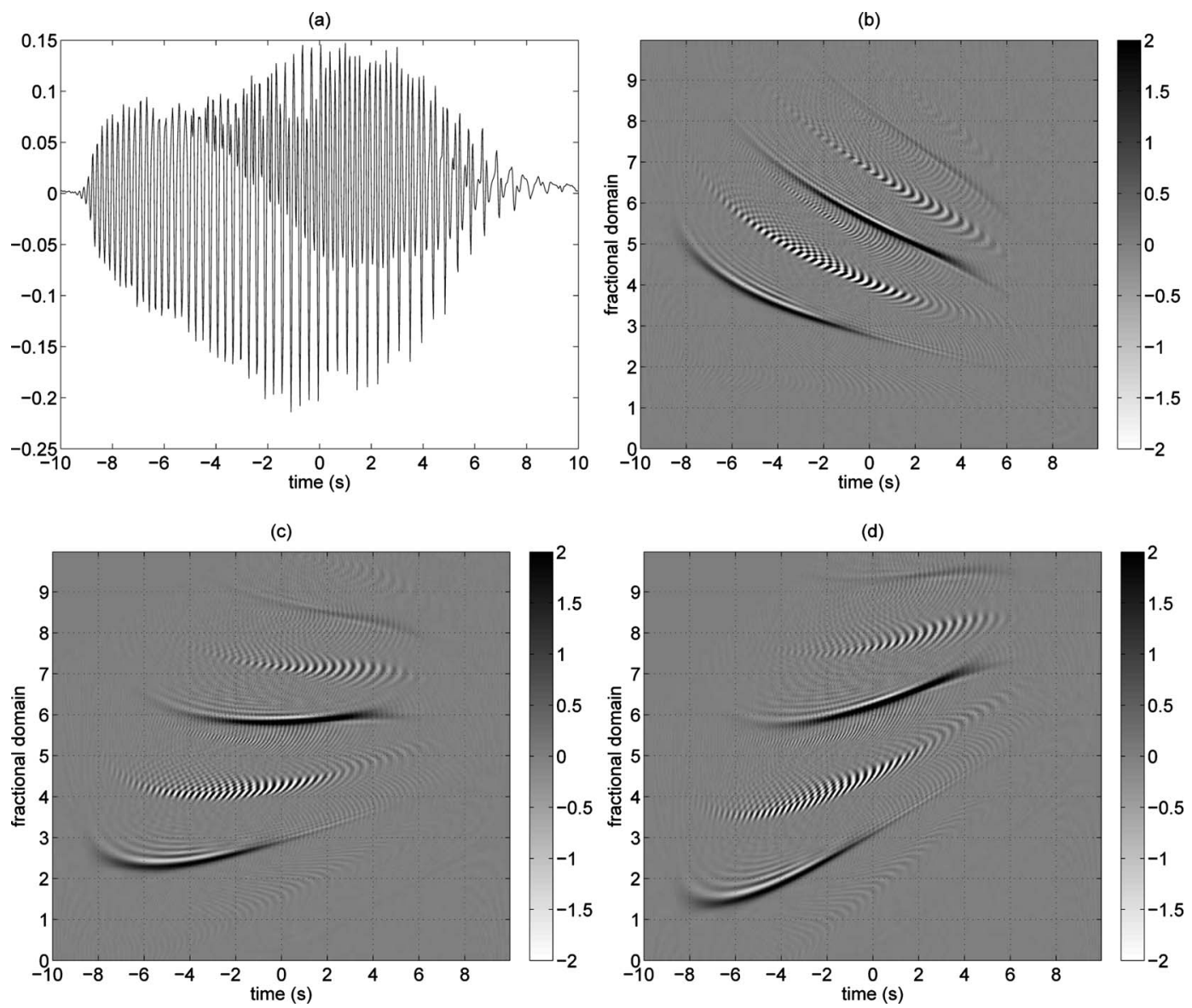

Fig. 5. (a) $2.5 \mathrm{~ms}$ echolocation pulse emitted by a large brown bat, eptesicus fuscus. The JFSRs at joint fractional Fourier domains with orders (b) $\left(a_{1}, a_{2}\right)=(0,1),(\mathrm{c})\left(a_{1}, a_{2}\right)=(0,1.2)$, (d) $\left(a_{1}, a_{2}\right)=(0,1.3)$. 
(a)

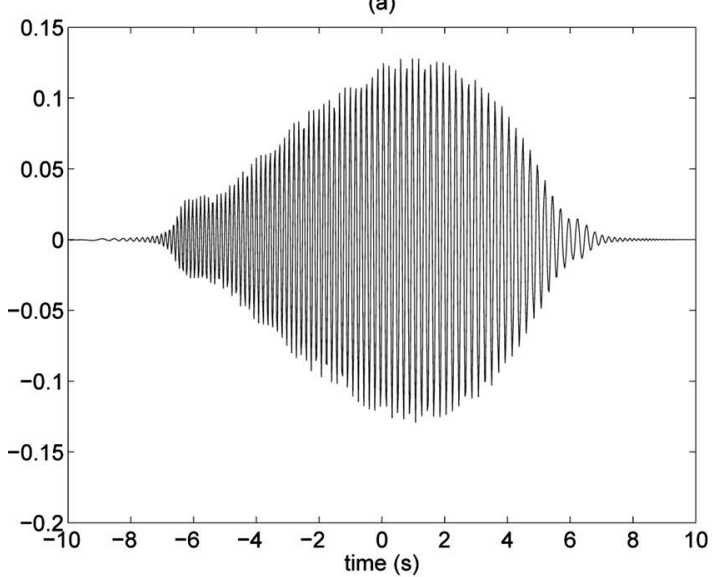

(c)

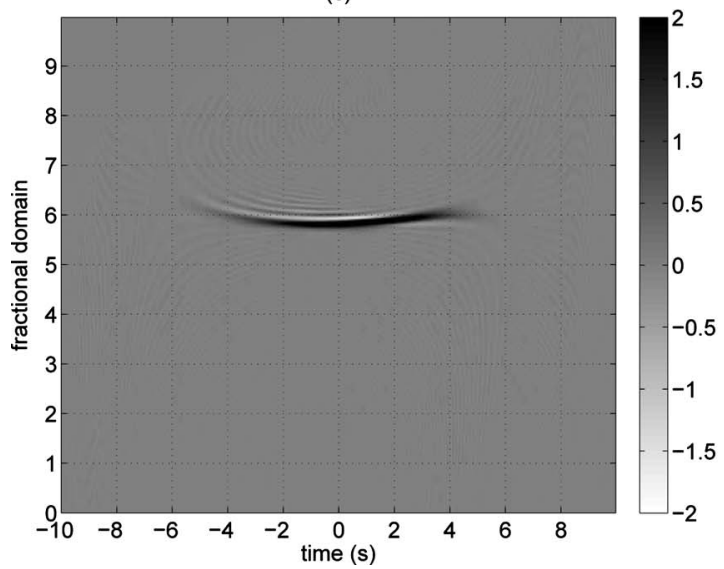

(b)

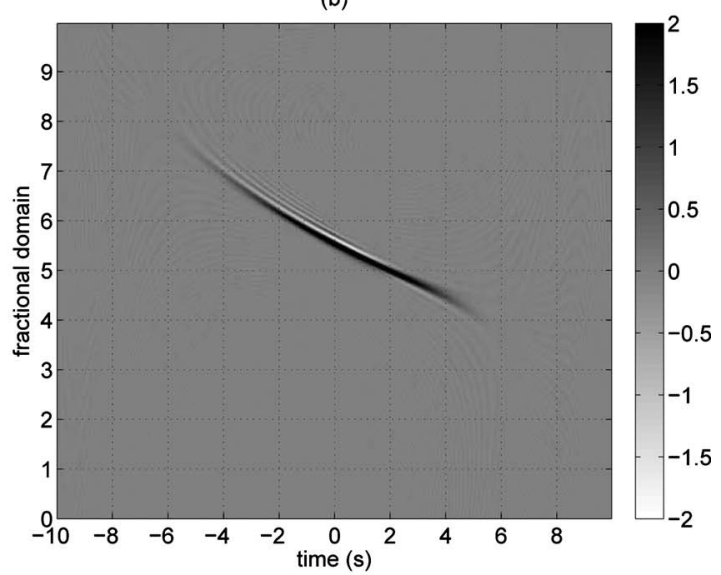

(d)

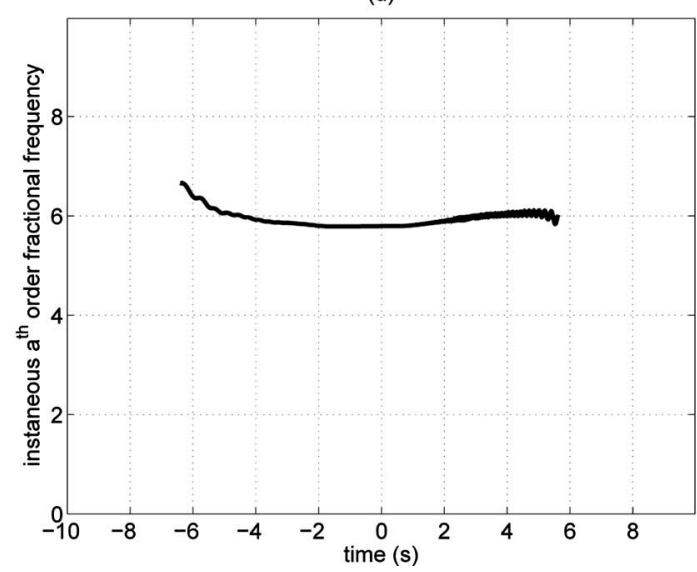

Fig. 6. (a) One of the components extracted from the bat signal. (b) The WD of the component and (c) its JFSR corresponding to the fractional Fourier order pairs of $(0,1.2)$. (d) The computed instantaneous frequency from the JFSR shown in (c).

$$
f_{a_{1}, a_{2}}(u)=\frac{\int_{-\infty}^{\infty} v W_{a_{1}, a_{2}}(u, v) \mathrm{d} v}{\int_{-\infty}^{\infty} W_{a_{1}, a_{2}}(u, v) \mathrm{d} v}
$$

which is the center of mass of the JFSR along the $v$ axis. The plot Fig. 6(d) shows the computed instantaneous frequency from the JFSR shown in Fig. 6(c). Essentially, this is the $a_{2}$ th-order fractional frequency content in the signal $x_{a_{1}}(u)$.

\section{CONCLUSIONS}

A joint fractional domain signal representation is developed using the energy density interpretation of the WD on the time-frequency plane. The $(u, v)$ axes defining the joint representation are chosen as the $\mathbf{a}=\left(a_{1}, a_{2}\right)$ th-order fractional Fourier domains. The distribution is designed so that its projections onto the $u$ and $v$ axes gives the modulus square of the fractional Fourier transform of signals at the corresponding orders $a_{1}$ and $a_{2}$ as $\left|x_{a_{1}}(t)\right|^{2}$ and $\left|x_{a_{2}}(t)\right|^{2}$, respectively. It is shown that the distribution $E_{x}^{\mathbf{a}}(u, v)$ depends on the WD through a coordinate transformation. Therefore, the JFSR is a real-valued distribution, too. The overall integral of the JFSR on the $(u, v)$ plane gives the total energy of the signal. In this paper, as part of the novel results, oblique projections of the JFSR is also derived, and a fast computation algorithm designed for the computation of arbitrary radial slices of the WD in [15] is extended to the computation of the JFSR. The JFSRs of various signals at various fractionally ordered domains are presented, and the localization of the signal components are compared.

The JFSR is not analyzed in the framework of the familiar Cohen's class. Therefore, its introduction to the nonstationary signal processing will bring new insights into the design, filtering, analysis, and synthesis of signals.

\section{ACKNOWLEDGMENTS}

We thank Curtis Condon, Ken White, and Al Feng of the Beckman Institute of the University of Illinois for the bat data and for permission to use it in this paper.

\section{REFERENCES}

1. F. Hlawatsch and G. F. Boudreaux-Bartels, "Linear and quadratic time-frequency signal representations," IEEE Signal Process. Mag. 9, 21-67 (1992).

2. L. Cohen, Time-Frequency Analysis (Prentice Hall, 1995).

3. S. Mann and S. Haykin, "The chirplet transform: physical 
considerations," IEEE Trans. Signal Process. 43, 2745-2761 (1995).

4. R. G. Baraniuk and D. L. Jones, "Matrix formulation of the chirplet transform," IEEE Trans. Signal Process. 44, 3129-3135 (1996).

5. R. G. Baraniuk, "Beyond time-frequency analysis: energy density in one and many dimensions," IEEE Trans. Signal Process. 46, 2305-2314 (1998).

6. O. Akay and G. F. Boudreaux-Bartels, "Joint fractional signal representations,” J. Franklin Inst. 337, 365-378 (2000).

7. L. B. Almedia, "The fractional Fourier transform and timefrequency representations," IEEE Trans. Signal Process. 42, 3084-3091 (1994).

8. H. Ozaktas and O. Aytür, "Fractional Fourier domains," Signal Process. 46, 119-124 (1995).

9. H. M. Ozaktas, Z. Zalevsky, and M. A. Kutay, The Fractional Fourier Transform with Applications in Optics and Signal Processing (Wiley, 2000).

10. H. M. Ozaktas, B. Barshan, D. Mendlovic, and L. Onural, "Convolution, filtering, and multiplexing in fractional Fourier domains and their relation to chirp and wavelet transforms," J. Opt. Soc. Am. A 11, 547-559 (1994).

11. B. Barshan, M. A. Kutay, and H. M. Ozaktas, "Optimal filtering with linear canonical transformations," Opt. Commun. 135, 32-36 (1997).

12. M. A. Kutay, H. M. Ozaktas, O. Arikan, and L. Onural, "Optimal filtering in fractional Fourier domains," IEEE Trans. Signal Process. 45, 1129-1143 (1997).

13. M. A. Kutay and H. M. Ozaktas, "Optimal image restoration with the fractional Fourier transform," J. Opt. Soc. Am. A 15, 825-833 (1998).

14. M. F. Erden and H. M. Ozaktas, "Synthesis of general linear systems with repeated filtering in consecutive fractional Fourier domains," J. Opt. Soc. Am. A 15, 1647-1657 (1998).

15. A. K. Özdemir and O. Arikan, "Efficient computation of the ambiguity function and the Wigner distribution on arbitrary line segments," IEEE Trans. Signal Process. 49, 381-393 (2001).

16. L. Durak and O. Arikan, "Short-time Fourier transform: two fundamental properties and an optimal implementation," IEEE Trans. Signal Process. 51, 1231-1242 (2003).

17. V. Namias, "The fractional order Fourier transform and its application to quantum mechanics," J. Inst. Math. Appl. 25, 241-265 (1980).

18. L. M. Bernardo and O. D. D. Soares, "Fractional Fourier transform and optical systems," Opt. Commun. 110, 517-522 (1994).

19. H. M. Ozaktas and D. Mendlovic, "Fractional Fourier optics," J. Opt. Soc. Am. A 12, 743-751 (1995).

20. O. Aytür and H. Ozaktas, "Non-orthogonal domains in phase space of quantum optics and their relation to fractional Fourier transform," Opt. Commun. 120, 166-170 (1995).

21. A. W. Lohmann and B. H. Soffer, "Relationships between the Radon-Wigner and fractional Fourier transforms," J. Opt. Soc. Am. A 11, 1798-1801 (1994).

22. S. Shinde and V. M. Gadre, "An uncertainty principle for real signals in the fractional Fourier transform domain," IEEE Trans. Signal Process. 49, 2545-2548 (2001).

23. H. M. Ozaktas, O. Arikan, M. A. Kutay, and G. Bozdagi, "Digital computation of the fractional Fourier transform," IEEE Trans. Signal Process. 44, 2141-2150 (1996).

24. L. R. Rabiner, R. W. Schafer, and C. M. Rader, "The chirp $z$-transform algorithm and its applications," Bell Syst. Tech. J. 48, 1249-1292 (1969).

25. A. K. Ozdemir, S. Karakas, E. D. Cakmak, D. I. Tufekci, and O. Arikan, "Time-frequency component analyzer and its application to analysis of brains oscillatory activity," J. Neurosci. Methods 145, 107-125 (2005).

26. http://www.dsp.rice.edu/software/TFA/RGK/BAT/ batsig.bin.Z. 